\title{
Infectious ACS $\mid$ Diseases
}

Subscriber access provided by Caltech Library

\section{Article}

\section{Switchable membrane remodeling and antifungal defense by metamorphic chemokine XCL1}

Acacia F Dishman, Michelle W. Lee, Jaime de Anda, Ernest Y. Lee, Jie He, Anna Huppler, Gerard Wong, and Brian F. Volkman

ACS Infect. Dis., Just Accepted Manuscript • DOI: 10.1021/acsinfecdis.0c00011 • Publication Date (Web): 03 Apr 2020

Downloaded from pubs.acs.org on April 3, 2020

\section{Just Accepted}

"Just Accepted" manuscripts have been peer-reviewed and accepted for publication. They are posted online prior to technical editing, formatting for publication and author proofing. The American Chemical Society provides "Just Accepted" as a service to the research community to expedite the dissemination of scientific material as soon as possible after acceptance. "Just Accepted" manuscripts appear in full in PDF format accompanied by an HTML abstract. "Just Accepted" manuscripts have been fully peer reviewed, but should not be considered the official version of record. They are citable by the Digital Object Identifier (DOI®). "Just Accepted" is an optional service offered to authors. Therefore, the "Just Accepted" Web site may not include all articles that will be published in the journal. After a manuscript is technically edited and formatted, it will be removed from the "Just Accepted" Web site and published as an ASAP article. Note that technical editing may introduce minor changes to the manuscript text and/or graphics which could affect content, and all legal disclaimers and ethical guidelines that apply to the journal pertain. ACS cannot be held responsible for errors or consequences arising from the use of information contained in these "Just Accepted" manuscripts. 

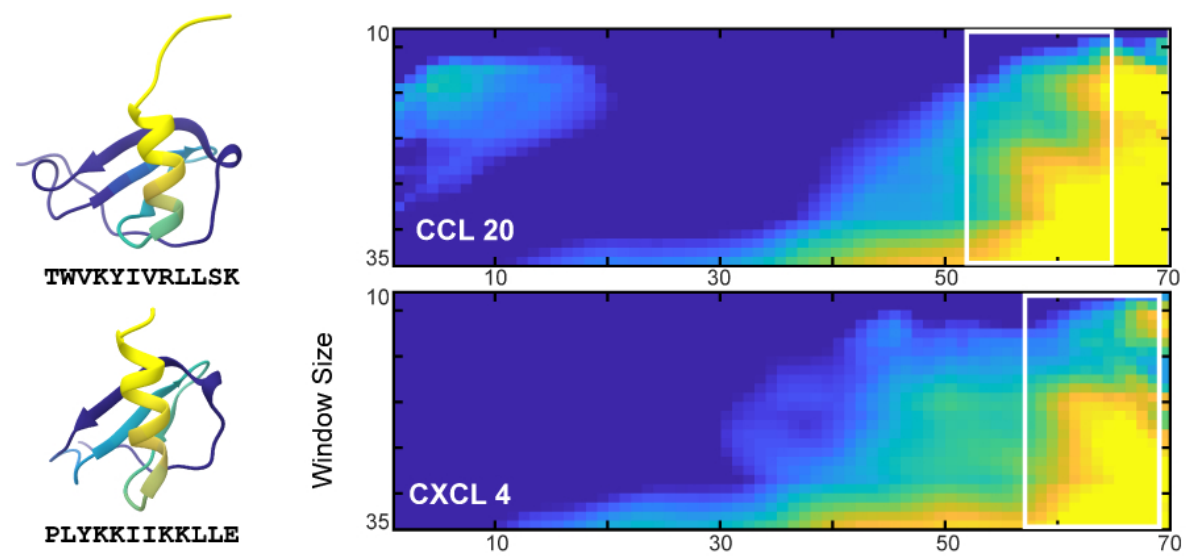

PLYKKIIKKLLE

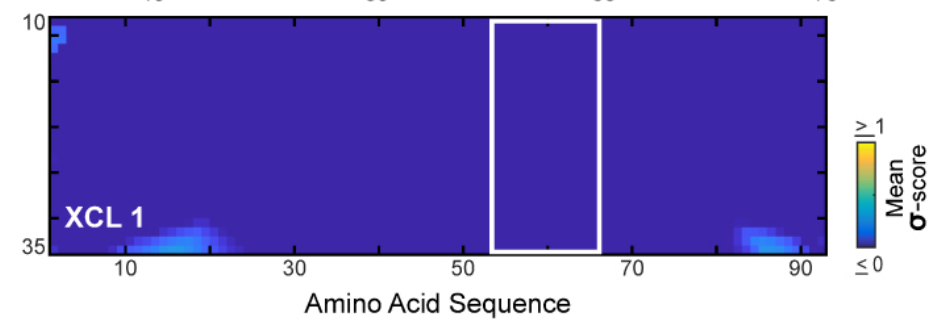

Figure 1.Machine learning based analysis indicates that XCL1's a-helix has drastically reduced levels of membrane remodeling activity compared to those of antimicrobial chemokines CCL20 and CXCL4. At left, the mean $\sigma$-score for a window size of 30 are projected on the 3D structures of CCL20, CXCL4, and XCL1.

Sequences of the helices are shown below each cartoon. At right, each chemokine's full sequence is visualized on a 2D probability map, with helices highlighted by a white box. $X$ axis, amino acid sequence. $Y$ axis, window size. Color, mean $\sigma$-score.

\section{$83 \times 56 \mathrm{~mm}(300 \times 300 \mathrm{DPI})$}




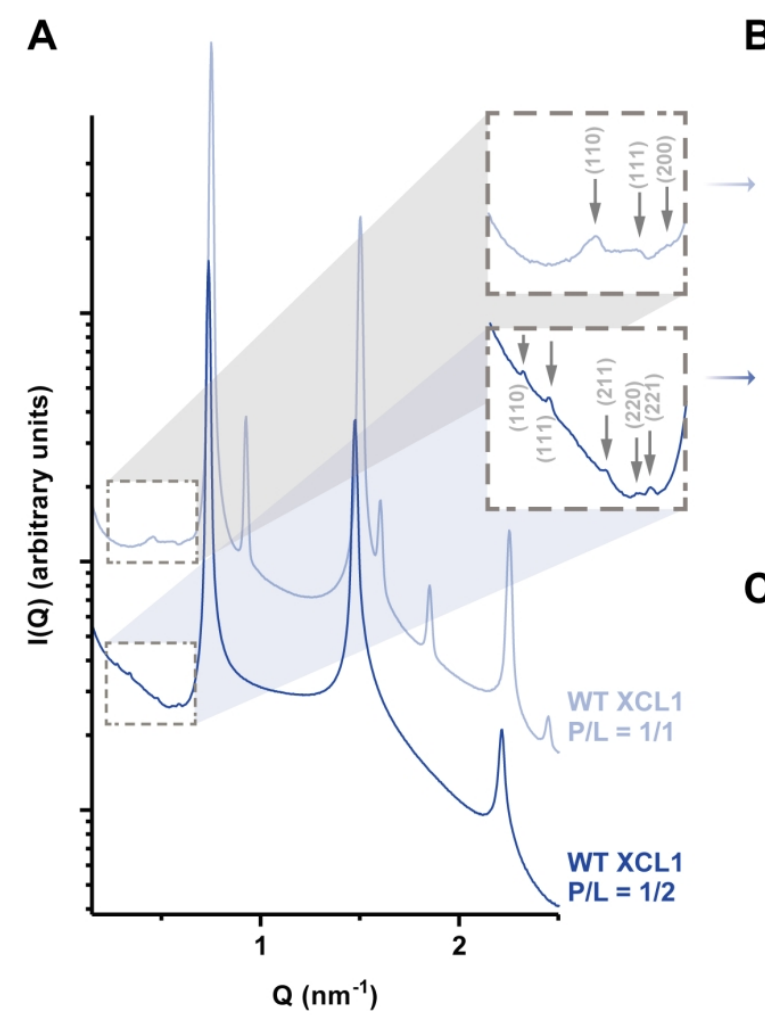

B

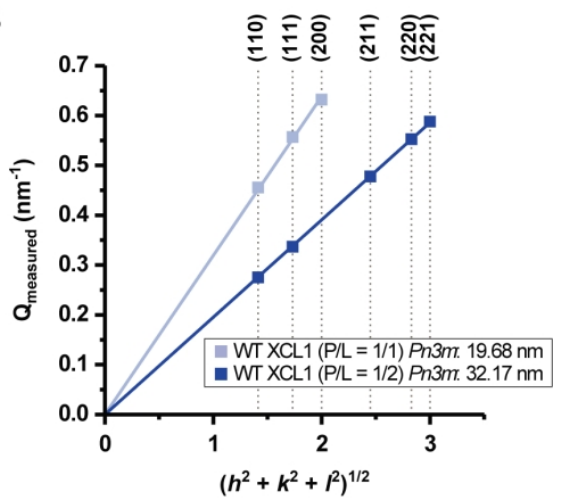

C

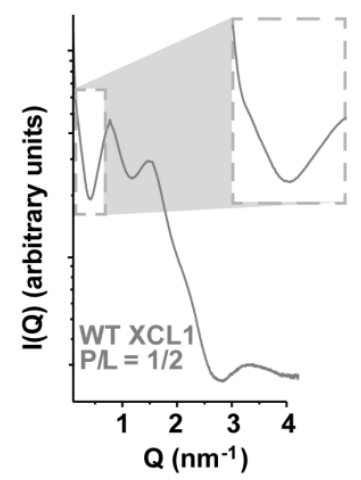

Figure 2.XCL1 induces Negative Gaussian Curvature in model bacterial membranes, but not model mammalian membranes. (A) SAXS spectra for XCL1 incubated with SUVs that have bacteria-like membrane compositions (DOPG/DOPE 20/80) at a P/L ratio of $1 / 1$ (light blue) and $1 / 2$ (dark blue). To facilitate visualization, spectra have been manually offset in the vertical direction by scaling each trace by a multiplicative factor. Spectra show characteristic Q peaks of NGC-rich Pn3m cubic phases (dashed grey boxes), which are indexed in (B). (C) SAXS spectra for XCL1 incubated with model mammalian SUVs (DOPS/DOPE/DOPC 20/40/40) in a P/L ratio of $1 / 2$ show no detectable Q peaks (dashed grey box).

$167 \times 129 \mathrm{~mm}(300 \times 300 \mathrm{DPI})$ 
Figure 3. Structural and functional features of XCL1 variants tested for membrane remodeling activity. (A) Panel of seven XCL1 variants tested in this study. WT XCL1 can access a $\beta$-sheet structure and chemokinelike $a-\beta$ structure and is thought to interconvert between the two via complete unfolding. W55D can access the $\beta$-sheet and unfolded state, but not the chemokine structure. CC5 can access only the $\beta$-sheet structure. XCL1 1-72 is a truncated version of WT XCL1 lacking the extended C-terminal tail (residues 73-93). XCL1

R23A and R43A are variants of XCL1 with reduced affinity for glycosaminoglycans which preferentially occupy the chemokine structure but can access the unfolded and all- $\beta$ structures. CCO can access only the unfolded state. CC3 can access only the chemokine structure. (B) Published values for WT XCL1 and select XCL1 structural variants' minimum effective concentrations (MEC, $\mu \mathrm{M}$ ) against E. Coli BL21 cells.12 CC3, an XCL1 structural variant locked in the chemokine structure, is notably less active.

$$
79 \times 156 \mathrm{~mm}(300 \times 300 \text { DPI) }
$$




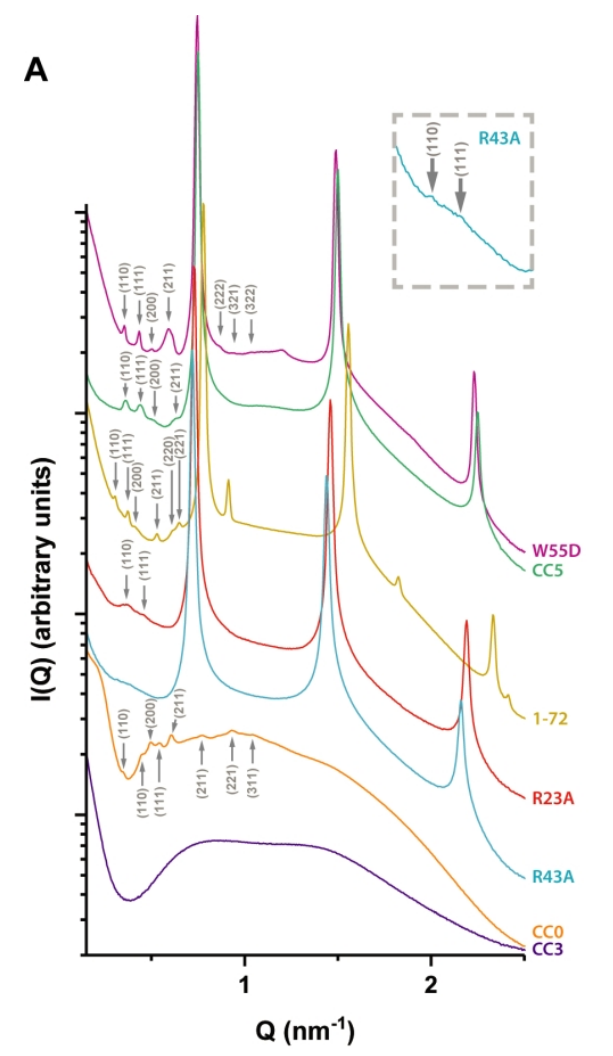

\section{B}

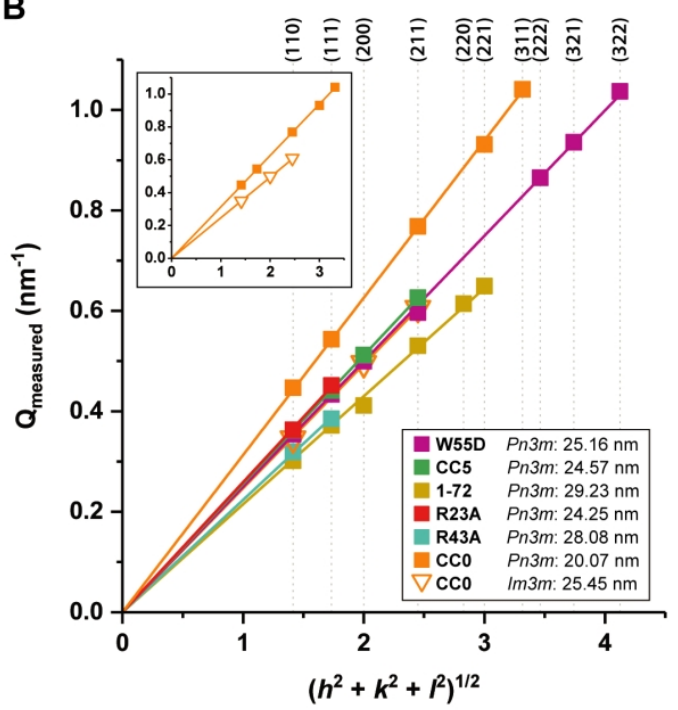

C

\begin{tabular}{c|c|c|c|c|c|c|c|c} 
& XCL1 & CC3 & CC5 & W55D & CC0 & $1-72$ & R23A & R43A \\
\hline $\begin{array}{c}\text { PG/PE } \\
\text { 20/80 } \\
\text { (bacterial) }\end{array}$ & $\checkmark$ & $x$ & $\checkmark$ & $\checkmark$ & $\checkmark$ & $\checkmark$ & $\checkmark$ & $\checkmark$ \\
\hline $\begin{array}{c}\text { PS/PE/PC } \\
\text { 20/40/40 } \\
\text { (mammalian) }\end{array}$ & $x$ & $x$ & $x$ & $x$ & $x$ & $x$ & $x$ & $x$
\end{tabular}

Figure 4. XCL1 requires access to its unfolded state or $\beta$-sheet structure, but not its C-terminal tail, for membrane remodeling. (A) SAXS spectra for model bacterial membranes (DOPG/DOPE 20/80) incubated with XCL1 structural variants. To facilitate visualization, spectra have been manually offset in the vertical direction by scaling each trace by a multiplicative factor. All variants except CC3, which is locked into the chemokine fold, induce NGC in model bacterial membranes. (B) Indexation plots for all variants shown in (A) that induce NGC. Inset: isolated indexation plot for CCO to provide additional clarity of the coexisting Pn3m and Im3m cubic phases. (C) Summary of NGC induction by each variant in model bacterial and mammalian membranes. Check marks indicate induction of NGC.

$174 \times 138 \mathrm{~mm}(300 \times 300 \mathrm{DPI})$ 


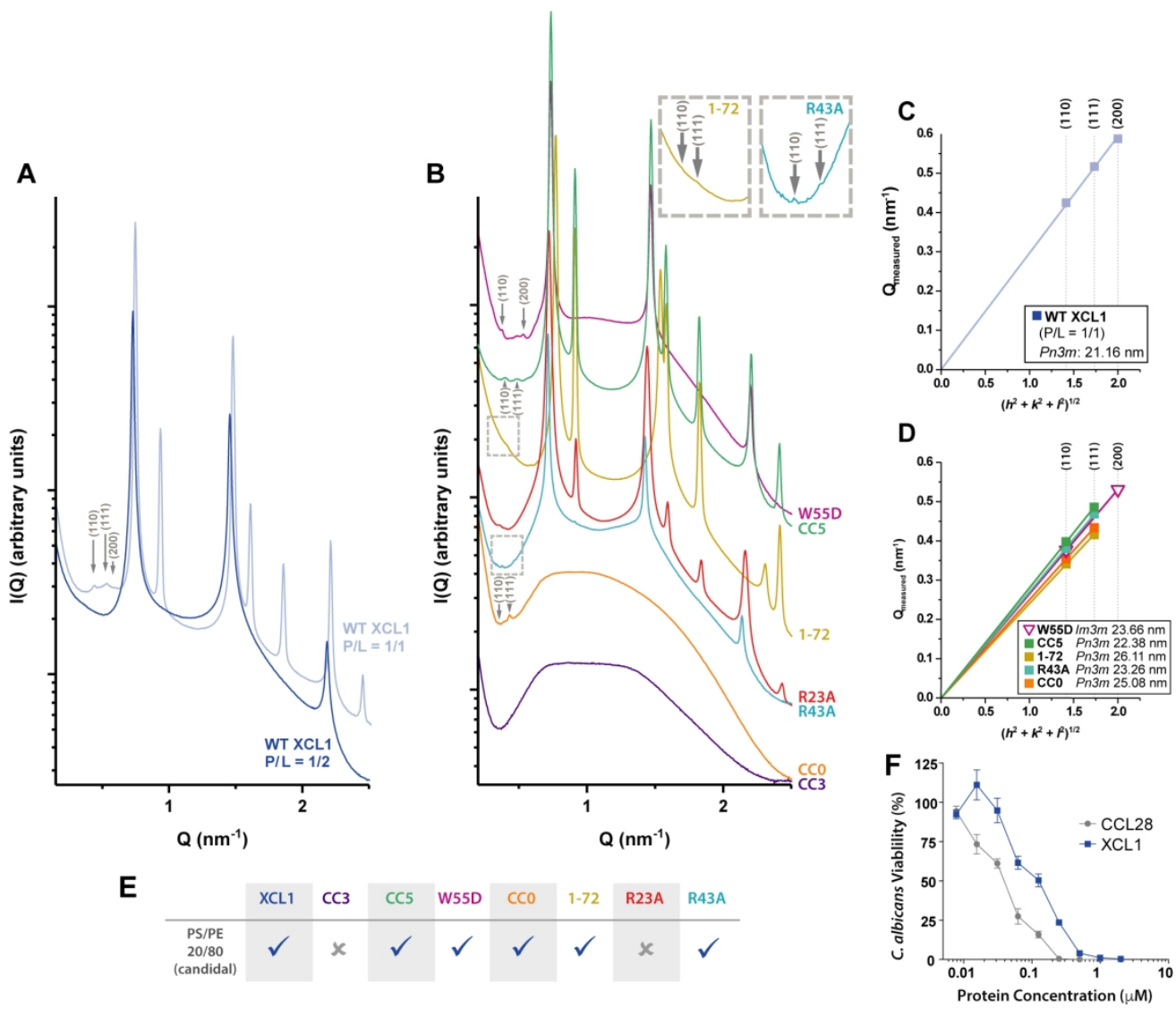

Figure 5.XCL1 induces NGC in model fungal membranes in a structurally dependent manner and can kill Candida in vitro. (A, B) SAXS spectra for model fungal membranes (DOPS/DOPE 20/80) incubated with XCL1 and the panel of XCL1 structural variants described in Figure 3. To facilitate visualization, spectra have been manually offset in the vertical direction by scaling each trace by a multiplicative factor. (C, D) Indexation plots for the spectra in A and B. (E) Summary of NGC induction by each XCL1 variant in model fungal membranes. (F) In vitro Candida killing assay data for XCL1 (blue) and CCL28 (positive control, grey).

$173 \times 180 \mathrm{~mm}(300 \times 300 \mathrm{DPI})$ 


\section{Switchable membrane remodeling and antifungal defense by metamorphic chemokine XCL1}

Acacia F. Dishman ${ }^{+}$and Michelle W. Lee ${ }^{+}$, Jaime de Anda ${ }^{2}$, Ernest Y. Lee', 3, Jie He4, Anna R. Huppler, Gerard C. L. Wong ${ }^{2}$ and Brian F. Volkman ${ }^{1}$ *

${ }^{1}$ Department of Biochemistry, Medical College of Wisconsin, Milwaukee, WI 53226 USA

${ }^{2}$ Department of Bioengineering, University of Los Angeles, Los Angeles, CA 90095, USA

${ }^{3}$ UCLA-Caltech Medical Scientist Training Program, David Geffen School of Medicine at UCLA, Los Angeles, CA 90095, USA

${ }^{4}$ Department of Pediatrics, Medical College of Wisconsin, Milwaukee, WI 53226 USA

t These authors contributed equally to this work

*Corresponding Author: Brian F. Volkman, bvolkman@mcw.edu

KEYWORDS: metamorphic proteins, XCL1, chemokines, small-angle Xray scattering, antimicrobial, antifungal 
ABSTRACT

Antimicrobial peptides (AMPs) are a class of molecules which generally kill pathogens via preferential cell membrane disruption. Chemokines are a family of signaling proteins that direct immune cell migration and share a conserved $\alpha-\beta$ tertiary structure. Recently, it was found that a subset of chemokines can also function as AMPs, including CCL20, CXCL4, and XCL1. It is therefore surprising that machine learning based analysis predicts that CCL20 and CXCL4's $\alpha$-helices are membrane disruptive, while XCL1's helix is not. XCL1, however, is the only chemokine known to be a metamorphic protein which can interconvert reversibly between two distinct native structures (a $\beta$-sheet dimer and the $\alpha-\beta$ chemokine structure). Here, we investigate XCL1's antimicrobial mechanism of action with a focus on the role of metamorphic folding. We demonstrate that XCL1 is a molecular 'Swiss army knife' that can refold into different structures for distinct contextdependent functions: whereas the $\alpha-\beta$ chemokine structure controls cell migration by binding to G-Protein Coupled Receptors (GPCRs), 
we find using Small Angle X-ray Scattering (SAXS) that only the $\beta$ sheet and unfolded XCL1 structures can induce negative Gausian curvature in membranes, the type of curvature topologically required for membrane permeation. Moreover, the membrane remodeling activity of XCLI's $\beta$-sheet structure is strongly dependent on membrane composition: XCL1 selectively remodels bacterial model membranes but not mammalian model membranes. Interestingly, XCL1 also permeates fungal model membranes and exhibits anti-Candida activity in vitro, in contrast to the usual mode of antifungal defense which requires Th17 mediated cell-based responses. These observations suggest that metamorphic XCL1 is capable of a versatile multi-modal form of antimicrobial defense. 
Antimicrobial peptides (AMPs) are innate immune molecules that often exhibit a common antimicrobial mechanism of disrupting pathogen membranes, which leads to depolarization, leakage, and eventual cell death. ${ }^{1-2}$ Examples of AMPs include cathelicidins, defensins, bacteriocins, histatins, protegrins, and more recently, an increasing number of chemokines, which are more commonly thought of as signaling molecules. ${ }^{2-8}$ Among the human chemokines that exhibit antibacterial, antifungal, or antiviral properties are CCL209, $\operatorname{CXCL} 4^{10}, \operatorname{CCL} 28^{11}, \operatorname{CXCL} 17^{12}$, and XCL113. Chemokines are a family of 50 small, secreted proteins that direct cellular migration by binding to glycosaminoglycans (GAGs) to form concentration gradients, and by binding to G-Protein Coupled Receptors (GPCRS) to induce pro-migratory cellular changes. All members of the chemokine family share a conserved tertiary structure consisting of a three-stranded antiparallel $\beta$-sheet and C-terminal $\alpha$-helix.

XCL1 provides a unique opportunity to demonstrate in an unambiguous manner the relationship between sequence, secondary and tertiary structure, and distinct forms of immune activity in a chemokine. XCL1 is unique among chemokines because it is a metamorphic protein, meaning that it reversibly switches between two or more native structures. ${ }^{14-15}$ Thus, using XCL1 as a model system, it becomes possible to interrogate what happens to 
signaling activity or membrane remodeling activity when the organization of secondary structure changes without changing the amino acid content. While most chemokines bind to GAGs and GPCRs using a single structure, XCL1 partitions these roles between its native states, which are (1) the highly conserved chemokine fold that binds its target receptor XCR1, and (2) a novel four-stranded $\beta$-sheet dimer that binds to GAGs.16-17 of XCL1's two native conformations, the alternative $\beta$-sheet structure displays strong antibacterial activity. ${ }^{13}$

Here, we investigated XCLI's ability to remodel membranes like AMPs as a function of its available conformational states, since previous work has attributed the bactericidal activity of XCL1 to its ability to permeabilize bacterial membranes. ${ }^{13}$ We applied a recently-developed machine learning classifier capable of reliably predicting membrane-active $\alpha$-helical peptide sequences to several prototypical chemokines. ${ }^{18}$ The classifier identified the $\alpha$-helices of CCL20 and CXCL4 as motifs that impart membrane activity to these chemokines. Surprisingly, however, the cognate helix of XCL1 is not predicted to induce negative Gausian curvature (NGC) in membranes. We used synchrotron small angle Xray scattering (SAXS) to measure membrane curvature deformations induced by XCL1 and a panel of XCL1 structural variants in membranes modeling Escherichia coli, Candida albicans, and 
mammalian cells. We found that XCL1 generates NGC in E. coli-like model membranes in a manner that depends on its metamorphic structural state: The $\beta$-sheet structure exhibits strong remodeling activity whereas the $\alpha-\beta$ chemokine structure does not. Whereas antifungal defense usually requires clearance by Th17 mediated cell-based immune responses, we find that XCL1 induces NGC in Candida-like membranes via a structural motif distinct from classical $\alpha-\beta$ chemokines, and exhibits anti-candida activity in vitro. This suggests the possibility of a versatile multi-modal antifungal defense associated with this metamorphic chemokine.

\section{RESULTS AND DISCUSSION}

The presence or absence of membrane activity in different folded states of XCL1 is central to the manner in which different immune functions are programmed into this metamorphic chemokine. A large body of work has found that specific physicochemical properties, including positive net charge and amphipathicity, of AMPs contribute to their bactericidal activity by facilitating electrostatic and hydrophobic interactions between the peptide and cell membranes. ${ }^{19}$ These interactions result in membrane destabilization, which can manifest in pore formation ${ }^{20-23,}$ blebbing, ${ }^{24-25}$ and vesicle budding. ${ }^{26}$ A topological requirement shared by many membrane-permeation processes is the induction of 
negative Gaussian curvature (NGC) in target membranes. ${ }^{27}$ NGC, also known as saddle-splay curvature, can be seen in the hole of a transmembrane pore or the neck of a fusion pore: for a flat horizontal surface labeled by cardinal directions (north (N), east $(E)$, south (S), west (S)), NGC can be generated by deforming the surface upward in the $\mathrm{N}$ and $\mathrm{S}$ directions and downward in the $\mathrm{E}$ and W directions. A broad range of studies identified a strong correlation between the ability of AMPs, AMP mutants, and synthetic AMP analogues to disrupt bacterial membranes and their capacity to generate NGC in membranes ${ }^{19}, 28-34$.

\section{XCL1 lacks a membrane active $\alpha$-helix}

Many antimicrobial peptides are thought to destabilize phospholipid bilayers by inducing NGC, a topological feature of membrane pores and blebs ${ }^{19,28-34}$. Previously, we developed a machinelearning support vector machine (SVM) classifier trained to identify $\alpha$-helical peptide sequences with the ability to remodel membranes by generating negative Gaussian curvature ${ }^{18}$. In recent work, we have shown that even though the SVM was trained on short $\alpha$-helical peptide sequences, the classifier is able to identify the existence of membrane active segments within larger membrane active proteins ${ }^{35-36}$. This machine learning approach constitutes a new kind of bioinformatic analysis that is based on function rather than structural or sequence homology. We aimed to apply this 
approach to chemokines, by determining whether the ability to generate NGC can be found in the $\alpha$-helical domain of the characteristic $\alpha-\beta$ chemokine fold. We assessed differences between the sequences of XCL1, and two other antimicrobial chemokines, CXCL4 and CCL20, using the SVM classifier to predict membrane active peptide subdomains ${ }^{18}$. This sequence-based approach allows us to score individual amino acid segments and identify $\alpha$-helical (or disordered) regions that are potentially involved in membrane remodeling

We screened the chemokine sequences using a 'moving window' scan to isolate individual segments of $n$ amino acids. Each subsequence was scored by the SVM classifier with a $\sigma$-score corresponding to its probability to be membrane disruptive, P(+1). The mean $\sigma$-score for each amino acid position is an average of the scores for all the sequences in which that amino acid appears for a given window size $n$. A large positive $\sigma$-score $(\sigma>0.89)$ corresponds to a high probability of membrane activity (P(+1) > 0.95), while a negative $\sigma$-score $(\sigma<0)$ indicates a small probability of membrane activity $(P(+1)<0.50)$. The scores for each chemokine's full sequence are visualized on a 2D probability map for different window sizes (Figure 1). For CXCL4 and CCL20, only the $\alpha$-helical C-terminal regions were predicted to have membrane remodeling ability. In contrast, the entire XCL1 sequence 
scored poorly for membrane activity (Figure 1) even though XCL1 exhibits antimicrobial activity ${ }^{13}$. The individual $\alpha$-helical sequence for CCL20 scored slightly higher than the CXCL4 $\alpha$-helix (TWVKYIVRLLSK, $\sigma=1.0, \mathrm{P}=0.97 ; \mathrm{PLYKKIIKKLLE,} \sigma=0.72, \quad \mathrm{P}=0.92$, respectively), whereas the XCL1 $\alpha$-helix scored significantly lower (TWVRDVVRSMDRK, $\sigma=0.13, \quad \mathrm{P}=0.61$ ). Although the tertiary $\alpha-\beta$ structure is similar between the three chemokines (Figure 1), the machine-learning classifier approach was able to identify subtle differences in these sequences using its optimized physicochemical descriptors. The presence of a membrane active $\alpha$-helix in CXCL4 and CCL20 is consistent with the antimicrobial character of these chemokines in their native $\alpha-\beta$ form and provides contrast to understand the minimal antimicrobial activity of XCL1 in its $\alpha-\beta$ conformation, with a membrane inactive $\alpha$-helix. Given that the SVM classifier is optimized to predict membrane active $\alpha$-helical sequences and no membrane activity is identified in the XCL1 $\alpha-$ helix cognate to the helices in the other $\alpha-\beta$ chemokines examined, we hypothesize that membrane remodeling activity in XCL1 arises from its ability to switch into a $\beta$-sheet structure, since such membrane activity is not detectable by an $\alpha$-helical classifier. 


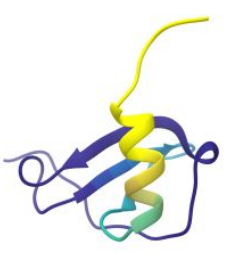

TWVKYIVRLLSK

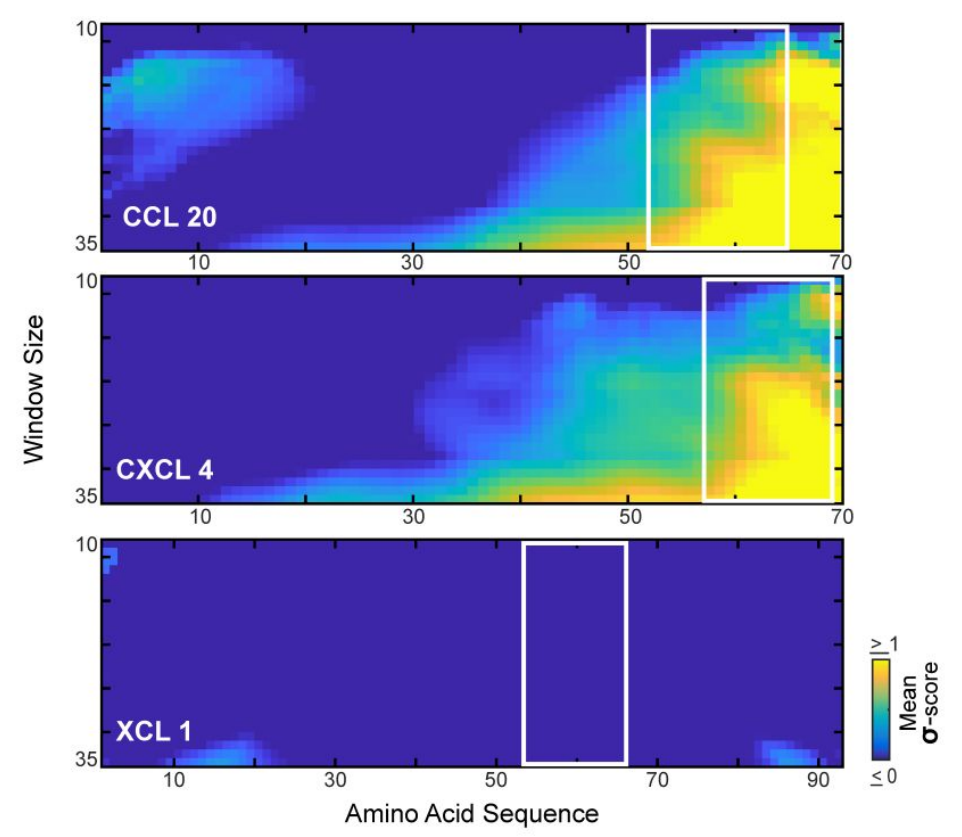

Figure 1.Machine learning based analysis indicates that XCL1's $\alpha$-helix has drastically reduced levels of membrane remodeling activity compared to those of antimicrobial chemokines CCL20 and CxCL4. At left, the mean $\sigma$-score for a window size of 30 are projected on the 3D structures of CCL20, CXCL4, and XCL1. Sequences of the helices are shown below each cartoon. At right, each chemokine's full sequence is visualized on a 2D probability map, with helices highlighted by a white box. $x$ axis, amino acid sequence. $Y$ axis, window size. Color, mean $\sigma$-score.

XCL1 induces negative Gaussian curvature necessary for membrane permeation in membranes modeling bacterial, but not mammalian cells 
To understand the specific biophysical mechanisms driving XCLl's ability to selectively kill bacteria, we employed synchrotron SAXS experiments to quantitatively characterize XCL1induced curvature deformations in model cell membranes. Although AMPs can kill microbes via a range of mechanisms, it has been recently shown that what AMPs have in common is their ability to generate NGC in microbial membranes, ${ }^{18}$ which is related to the ability to kill microbes through membrane disruptive modes such as pore formation and blebbing $27,30,37-38$.

Since selectivity of AMP-induced killing is thought to arise in part from the ability to recognize differences in membrane lipid composition between microbes and host cells, we compared the curvature deformations induced by XCL1 in two different membranes: one modeling E. coli membranes and one modeling mammalian cell membranes. Small unilamellar vesicles (SUVs) with lipid compositions mimicking that of $E$. coli membranes and mammalian cell membranes were incubated with XCL1, and the resulting crystalline structures were characterized using SAXS. Bacterial membranes were modeled using a lipid composition of 1,2-dioleoylsn-glycero-3-[phospho-rac-(1-glycerol)]/1,2-dioleoyl-sn-glycero3-phosphoethanolamine at a molar ratio of 20/80 (DOPG/DOPE 20/80). Mammalian cell membranes were modeled using a lipid composition of 1,2-dioleoyl-sn-glycero-3-phospho-L-serine/1,2-dioleoyl-snglycero-3-phosphoethanolamine/1,2-dioleoyl-sn-glycero-3- 
phosphocholine at a molar ratio of 20/40/40 (DOPS/DOPE/DOPC 20/40/40). XCL1 was incubated with the SUVs at protein/lipid charge ratios (P/L) of $1 / 2$ and $1 / 1$ (i.e. molar ratios of $1 / 85$ and 2/85, respectively).

XCL1 restructured E. coli-like membranes into phases rich in NGC (Figure 2A), while control samples of the SUVs alone displayed a broad characteristic feature consistent with the form factor expected for unilamellar vesicles (Supplemental Figure 1). SAXS spectra for SUVs with the E. coli-like membrane composition that were exposed to XCL1 exhibited correlation peaks with Q-ratios of $\sqrt{2}: \sqrt{ } 3: \sqrt{6}: \sqrt{ } 8: \sqrt{9}($ for $P / L=1 / 2$ ) and $\sqrt{2}: \sqrt{ } 3: \sqrt{ } 4($ for $P / L=1 / 1$ ), which index to $\operatorname{Pn} 3 m$ cubic phases with lattice parameters of $32.17 \mathrm{~nm}$ and $19.68 \mathrm{~nm}$, respectively (Figure 2A-B). Bicontinuous lipidic cubic phases (QII), such as the Pn $3 m$ "double diamond" cubic lattice seen here, are also commonly formed by prototypical AMPs with model bacterial membranes ${ }^{19},{ }^{28-32}$. A bicontinuous cubic phase consists of two nonintersecting aqueous regions that are separated by a lipid bilayer with NGC at every point on its surface. In a cubic phase, the average amount of Gaussian curvature, $K$, can be calculated using the equation $\left\langle K>=(2 \pi \chi) /\left(A_{0} a^{2}\right)\right.$, where the Euler characteristic, $\boldsymbol{x}$, and the surface area per unit cell, $A_{0}$, are constants specific to each cubic phase, and a is the lattice parameter. For Pn3m, $\chi=-2$ and $A_{0}=1.919 .{ }^{39}$ With the E. coli model 
membrane, XCL1 generated Pn 3 m cubic phases with $\left\langle K>\right.$ of $-6.33 \times 10^{-3}$ $\mathrm{nm}^{-2}($ for $\mathrm{P} / \mathrm{L}=1 / 2)$ and $-1.69 \times 10^{-2} \mathrm{~nm}^{-2}$ (for $\left.\mathrm{P} / \mathrm{L}=1 / 1\right)$, which in fact are of similar magnitude to NGC generated by many AMPs. ${ }^{30}$ In other words, our SAXS spectra indicate that XCL1 causes restructuring of model bacterial membranes into phases rich in NGC. Because this type of curvature is topologically required for membrane permeabilization mechanisms, these results suggest that XCL1 has the capacity to kill bacteria via direct, physical membrane disruption. In contrast, the SAXS profile of XCL1 mixed with SUVs mimicking mammalian cell membranes $(\mathrm{P} / \mathrm{L}=1 / 2)$ exhibited a set of correlation peaks with integral Q-ratios consistent with a lamellar $\left(L_{\alpha}\right)$ phase with a periodicity of 8.54 $\mathrm{nm}$, indicating the formation of multilamellar membrane stacks without significant curvature generation (Figure 2C). Similar to the E. Coli-like SUVs, the control samples of mammalian cell-like SUVs alone also exhibited the form factor expected of unilamellar vesicles.

Together, these results indicate that XCL1 can selectively kill bacteria via membrane disruption in a manner cognate with other known AMPs. Moreover, our data suggest that XCL1's selective antimicrobial activity arises from XCL1's ability to recognize differences between the membrane compositions of mammalian cells and bacteria. 
A

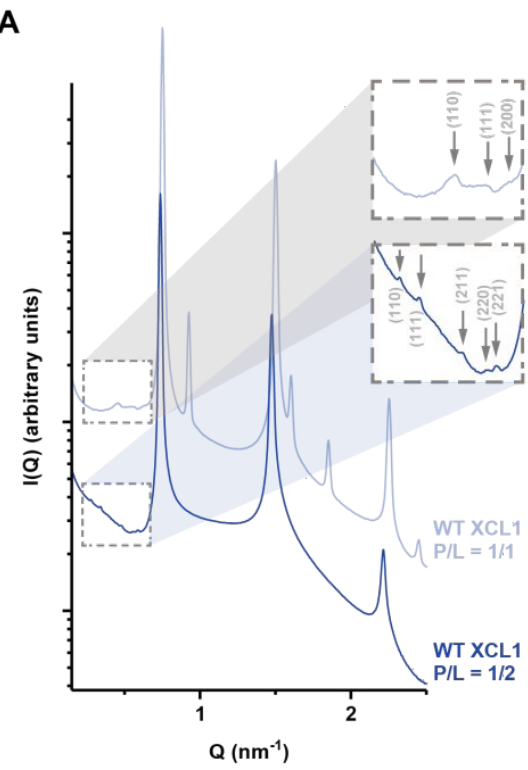

B

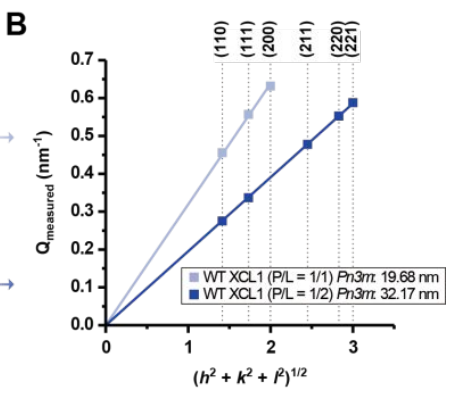

C

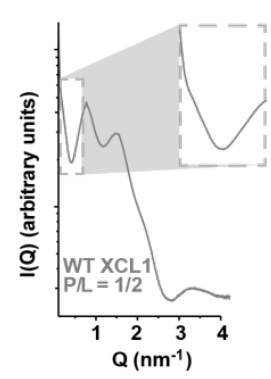

Figure 2.XCL1 induces Negative Gaussian Curvature in model bacterial membranes, but not model mammalian membranes. (A) SAXS spectra for XCL1 incubated with SUVs that have bacteria-like membrane compositions (DOPG/DOPE 20/80) at a $\mathrm{P} / \mathrm{L}$ ratio of $1 / 1$ (light blue) and $1 / 2$ (dark blue). To facilitate visualization, spectra have been manually offset in the vertical direction by scaling each trace by a multiplicative factor. Spectra show characteristic $Q$ peaks of NGC-rich Pn3m cubic phases (dashed grey boxes), which are indexed in (B). (C) SAXS spectra for XCL1 incubated with model mammalian SUVS (DOPS/DOPE/DOPC 20/40/40) in a $\mathrm{P} / \mathrm{L}$ ratio of $1 / 2$ show no detectable Q peaks (dashed grey box).

\section{A panel of structural XCL1 variants display differing membrane- disruptive activities in $E$. coli-like membranes}


XCL1 has been well studied, and a number of variants have been engineered to stabilize XCL1 in its chemokine, all- $\beta$, and unfolded states ${ }^{4-41}$. These variants are named CC3, CC5, and CC0 respectively. Additionally, an XCL1 point mutant, XCL1 W55D, destabilizes the hydrophobic core of XCL1's chemokine structure by replacing hydrophobic Trp55 with a negatively charged aspartate, and thus allows access to the all- $\beta$ and unfolded states, but not the chemokine structure ${ }^{17}$. Two other point mutants, XCL1 R23A and XCL1 R43A, shift XCL1's conformational equilibrium to favor its chemokine structure, in addition to dramatically diminishing XCL1's affinity for GAGS ${ }^{42-43}$. Finally, a truncated version of XCL1 (XCL1 residues $1-72$ ) is missing its extended C-terminal tail, without alteration to the structural equilibrium. The structural behaviors of these variants are illustrated in Figure $3 \mathrm{~A}$, and the antimicrobial activities of select variants against $E$. coli as measured by a radial diffusion assay ${ }^{13}$ are catalogued in Figure 3B. 


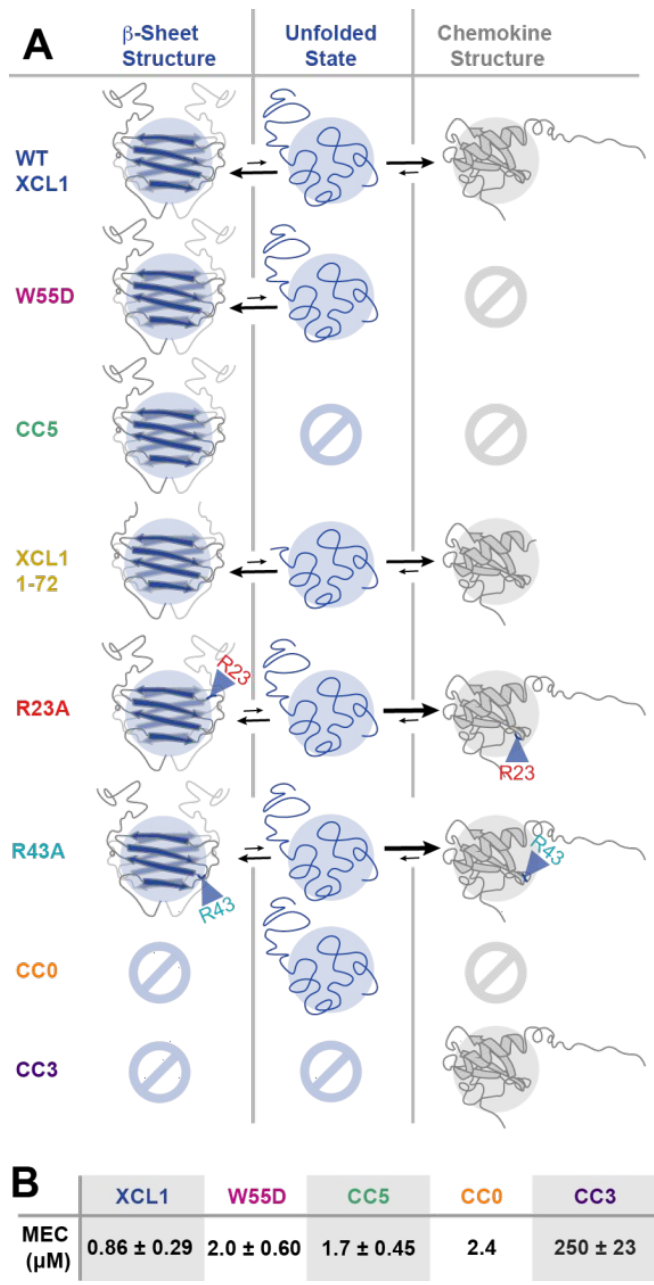

Figure 3. Structural and functional features of XCL1 variants tested for membrane remodeling activity. (A) Panel of seven XCL1 variants tested in this study. WT XCL1 can access a $\beta$-sheet structure and chemokine-like $\alpha-\beta$ structure and is thought to interconvert between the two via complete unfolding. W55D can access the $\beta$-sheet and unfolded state, but not the chemokine structure. CC5 can access only the $\beta$-sheet structure. XCL1 $1-72$ is a truncated version of WT XCL1 lacking the extended C-terminal tail (residues 73-93). XCL1 R23A and R43A are variants of XCL1 with reduced affinity for 
glycosaminoglycans which preferentially occupy the chemokine structure but can access the unfolded and all- $\beta$ structures. Cc0 can access only the unfolded state. CC3 can access only the chemokine structure. (B) Published values for WT XCL1 and select XCL1 structural variants' minimum effective concentrations (MEC, $\mu \mathrm{M}$ ) against $E$. Coli BL21 cells. ${ }^{13}$ CC3, an XCL1 structural variant locked in the chemokine structure, is notably less active.

To determine which specific structural features are influential in XCLI's antibacterial activity, we used synchrotron SAXS to systematically examine each of these seven variants' membrane disruption capabilities in SUVs with lipid compositions that mimic those of $E$. coli and mammalian cell membranes.

We found that CC5 (locked $\beta$-sheet structure), cC0 (unfolded variant), and W55D (accesses unfolded state and $\beta$-sheet structure) are all capable of generating NGC in E. coli-like membranes, in a manner similar to that of WT XCL1. The SAXS spectra of the SUVs after exposure to these proteins revealed the formation of NGCrich cubic phases (Figure 4A). For W55D and CC5 at P/L = 1/2, correlation peaks indexed to a Pn3m phase with a lattice parameter of $25.16 \mathrm{~nm}$ and $24.57 \mathrm{~nm}$, respectively (Figure 4B). For CCO at P/L $=1 / 2$, we observed a coexistence of Pn3m and Im3m cubic phases, the latter of which is defined by $\chi=-4$ and $A_{0}=2.345 .39$ The 
respective lattice parameters of these coexisting cubic phases were $20.07 \mathrm{~nm}$ and $25.45 \mathrm{~nm}$, which had a ratio close to the Bonnet ratio of 1.279 , indicating that the two cubic phases are near equilibrium with the amount of curvature being balanced across both.

SAXS spectrum from exposure of CC3 to E. coli-like membranes $(\mathrm{P} / \mathrm{L}=1 / 2)$ showed a broad feature that is consistent with the form factor of unilamellar vesicles and resembled the spectrum of the control SUVs, indicating that NGC was not induced and that the chemokine conformation does not effectively disrupt membranes at this $\mathrm{P} / \mathrm{L}$ ratio (Figure 4A). In other words, XCL1 requires access to its $\beta$-sheet structure or unfolded state in order to readily function as an antimicrobial agent with the mechanism of membrane permeation.

XCL1 R23A and XCL1 R43A also restructured E. coli-like SUVs $(\mathrm{P} / \mathrm{L}=1 / 2)$ into Pn3m cubic phases, as shown in Figure 4A, B. This indicates that despite their loss of GAG-binding affinity, these variants retain the ability to induce membrane disruption in $E$. coli-like membranes, disentangling XCLl's GAG-binding activity from its antimicrobial function. Compared with WT XCL1, the structural equilibria of these variants are shifted to favor the chemokine fold ${ }^{42,44}$. Nonetheless, they are still able to induce NGC in model bacterial membranes. In conjunction with the results from CC3, CCO, W55D, and CC5, this suggests that access to the unfolded 
and $\beta$-sheet structures is critical for membrane-disruptive function, and that proteins can remain highly effective as membrane disruptors even when these structures are less highly populated than in WT XCL1.

XCL1 1-72 (tail-less variant) was also able to induce NGC in E. coli-like membranes, as demonstrated by the generation of a Pn $3 m$ phase at $\mathrm{P} / \mathrm{L}=1 / 2$, indicating that $\mathrm{XCL1}$ 's extended $\mathrm{C}$-terminal tail (residues 73-93) is not required for membrane-disruptive activity. No XCL1 variants induced NGC in mammalian cell-like membranes even when structural and GAG-binding profiles are altered (Supplemental Figure 3).

The results described above indicate that XCL1 functions as a molecular 'swiss army knife' metamorph that partitions distinct, context-dependent functions into its different folded structures. While the $\alpha-\beta$ chemokine structure binds and activates its cognate GPCR XCR1, only the $\beta$-sheet and unfolded XCL1 structures can induce NGC in membranes. It is interesting to compare these structural tendencies of XCL1 to recently studied metaphilic peptides, which have an architecture that allow sidechains to re-organize in response to different environments ${ }^{33-34}$. In the case of XCL1, such functional changes are made possible by changes in secondary structure. 


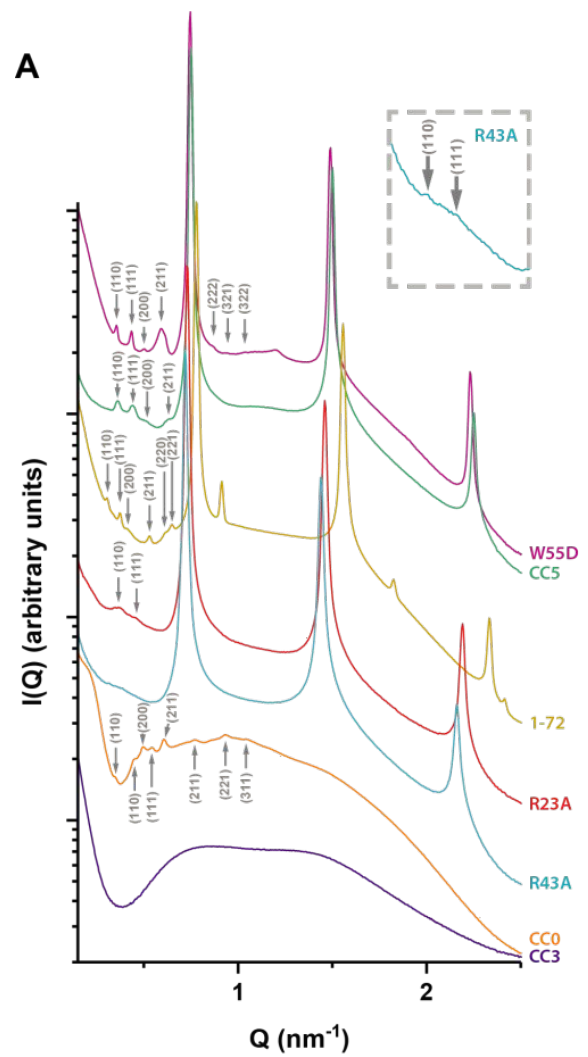

B

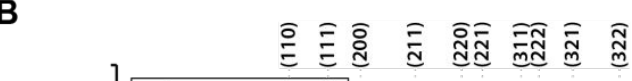

Figure 4. XCL1 requires access to its unfolded state or $\beta$-sheet structure, but not its C-terminal tail, for membrane remodeling. (A) SAXS spectra for model bacterial membranes (DOPG/DOPE 20/80) incubated with XCL1 structural variants. To facilitate visualization, spectra have been manually offset in the vertical direction by scaling each trace by a multiplicative factor. All variants except CC3, which is locked into the chemokine fold, induce NGC in model bacterial membranes. (B) Indexation plots for all variants shown in (A) that induce NGC. Inset: isolated indexation plot for CCO to provide additional clarity of the coexisting Pn3m and Im3m cubic phases. (C) Summary of NGC 
induction by each variant in model bacterial and mammalian membranes. Check marks indicate induction of NGC.

\title{
SAXS reveals XCL1's ability to induce NGC in a conformation- dependent dependent manner in Candida-like artificial membrane compositions
}

\begin{abstract}
Since fungi are eukaryotes, fungal membranes are more similar to mammalian membranes than bacterial membranes, and discrimination is often a challenge. As the structural motif for generating NGC in XCL1 is different than that of other chemokines ( $\beta$-sheet rather than the $\alpha-\beta$ fold) we investigated whether XCL1 is
\end{abstract} able to disrupt Candida-like membranes. SUVs with a Candida-like membrane composition, 1,2-dioleoyl-sn-glycero-3-phospho-Lserine/1,2-dioleoyl-sn-glycero-3-phosphoethanolamine in a molar ratio of 20/80 (DOPS/DOPE 20/80), were incubated with XCL1 and the resulting membrane structures were characterized using synchrotron SAXS. As with other lipid-only control samples, the SAXS profile from DOPS/DOPE 20/80 SUVS exhibited a form factor that is consistent with unilamellar vesicles (Supplemental Figure 1). However, the lipid vesicles restructured when exposed to XCL1 (P/L $=1 / 1)$, as indicated by correlation peaks with ratios $\sqrt{2}: \sqrt{ } 3: \sqrt{ } 4$ that index to a $P n 3 m$ cubic phase with a lattice parameter of $21.16 \mathrm{~nm}$ and $<K>$ of $-1.46 \times 10^{-2} \mathrm{~nm}^{-2}$ ( Figure 5A, C). 
The seven XCL1 structural variants described earlier (CC3, CC0, CC5, XCL1 W55D, XCL1 R23A, XCL1 R43A, and XCL1 1-72) were also each incubated with Candida-like membranes and characterized. We found that the structural dependence observed in bacteria-like membranes was also consistent in Candida-like membranes, in which variants that could access the unfolded or $\beta$-sheet structure were able to generate NGC. SAXS spectra for CCO, CC5, and W55D showed correlation peaks with Q-ratios indicative of the presence of $P n 3 m$ and Im3m cubic lattices rich in NGC (Figure 5B,D). As with E. colilike membranes, CC3 was not able to generate NGC in Candida-like membranes. Interestingly, XCL1 R23A also did not generate NGC in these membranes (Figure 5B,D), deviating from its pattern of membrane-disruptive activity with bacteria-like membranes. However, XCL1 R43A and XCL1 1-72 both generated NGC in Candidalike SUVs, consistent with their activity with bacterial membranes (Figure 5B,D). Collectively, these data suggest that similar structure-function relationships hold for XCLl's activity with candidal membranes as for bacterial membranes, with a slight nuance at GAG binding residue Arg23.

\section{XCL1 exhibits antifungal activity against Candida}

Several human mucosal chemokines, including CCL2811, 45 , CXCL1712, and CCL209, kill Candida but it is not clear whether this is a general feature of chemokines. Moreover, given the metamorphic 
nature of XCL1, it is also not known whether antifungal activity can exist in a molecule whose structures are transiently occupied. Our SAXS data demonstrate that XCL1 induces structural rearrangement necessary for membrane disruption in Candida-like membranes, suggesting that XCL1 should exhibit antifungal activity. To investigate this hypothesis, we performed in vitro killing assays against $C$. albicans and found XCLl to be a potent anti-candidal agent, similar to CCL28, which has known anticandidal activity ${ }^{11}$ (Figure 5F). While CCL28 is a slightly more potent killer of $C$. albicans perhaps due to XCLl's structural interconversion, both proteins achieve complete killing at concentrations of $1 \mu \mathrm{M}$. These findings are in agreement with the previously identified correlations between NGC generation and membrane-disruptive AMP activity, 28, 30 including that of mammalian $\alpha$-defensins, which also have $\beta$-sheet-rich secondary structures. ${ }^{29}$ Moreover, antifungal defense in mammals usually requires clearance by Th17 mediated neutrophil-based responses: Surprisingly, we find that XCL1 induces membrane permeating NGC in Candida-like membranes via a structural motif distinct from classical $\alpha-\beta$ chemokines. This suggests the possibility of a multi-layered antifungal defense associated with this metamorphic chemokine. Interestingly, both XCLI and IL-17A are produced by NKT cells ${ }^{47-48}$ and are involved in antifungal defense ${ }^{49}$, suggesting a possible functional, synergistic relationship between the two molecules, as 
well as a possible mechanism by which NKT cells participate in host defense against fungi.
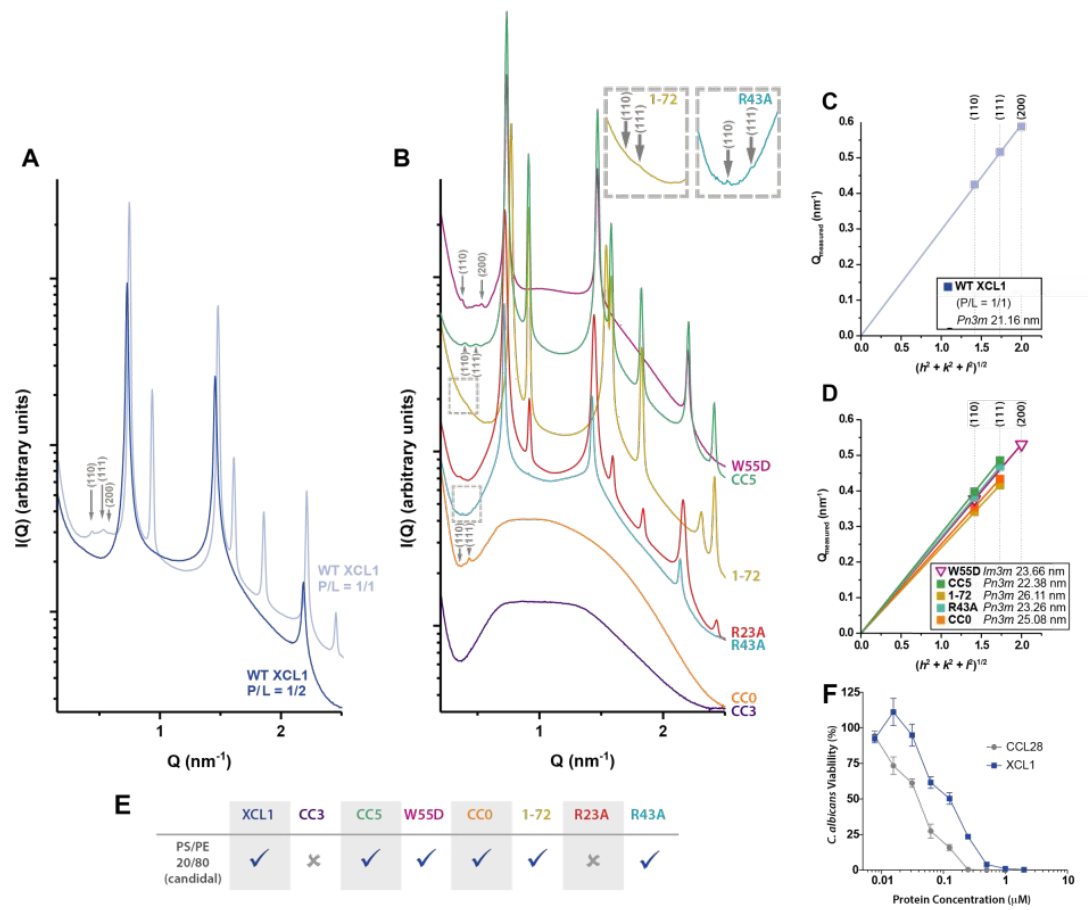

Figure 5.XCL1 induces NGC in model fungal membranes in a structurally dependent manner and can kill Candida in vitro. (A, B) SAXS spectra for model fungal membranes (DOPS/DOPE 20/80) incubated with XCL1 and the panel of XCL1 structural variants described in Figure 3. To facilitate visualization, spectra have been manually offset in the vertical direction by scaling each trace by a multiplicative factor. (C, D) Indexation plots for the spectra in A and B. (E) Summary of NGC induction by each XCL1 variant in model fungal membranes. (F) In vitro Candida killing assay data for XCL1 (blue) and CCL28 (positive control, grey). 


\section{CONCLUSIONS}

Many antimicrobial peptides, such as bacteriocins, defensins, histatins, protegrins, and chemokines, $2-6,12$ kill microbes via membrane disruption, for example by forming pores or blebs. For example, the human chemokine CXCL17 has been shown to kill E. Coli by inducing membrane disruption ${ }^{12}$. The generation of NGC in the target membrane is a topological requirement for the formation of these structures ${ }^{27}, 30,37-38$. We and others have previously detected antimicrobial activity for the human chemokine XCL1, a metamorphic chemokine with two native structures ${ }^{13}$. ${ }^{46}$. In this work, we find that XCL1 can selectively, physically disrupt bacterial and fungal membranes, and that XCLI's metamorphic folding allows for restriction of its membrane remodeling function to its $\beta$-sheet structure.

Our results suggest that XCLI's dynamic interconversion could provide the opportunity for switchable, context-dependent function. Because XCLI's $\alpha-\beta$ chemokine structure cannot induce membrane disruption and XCL1's $\beta$-sheet structure cannot bind to XCL1's cognate GPCR, shifts in equilibrium could tune XCL1 towards either membrane disruptive activity or receptor-binding activity in different environments, allowing for synergistic, contextdependent tuning of function. In fact, XCLl's structural 
equilibrium is known to shift to favor the $\beta$-sheet state in the presence of one of its binding partners, GAGs ${ }^{17}$. It is possible that the presence of microbial membranes can similarly shift XCL1's equilibrium toward the $\beta$-sheet structure, a hypothesis which will be explored in future studies.

In the present paradigm, proteins typically evolve to minimize structural frustration in the thermodynamically favored native state ${ }^{5-52}$. The structural and functional divergence observed for XCL1 has fundamental consequences for this classical picture of a folded protein: it is possible for the same sequence to encode different structures with distinct patterns of frustrations. We speculate that metamorphosis has evolved in XCL1 because it is associated with a gain of function that may enhance XCL1's 'fitness'53. Such a gain of function is discovered here: XCL1's conformation-specific, physical disruption of antibacterial and antifungal membranes illustrates how protein metamorphosis can allow for adjustability of function via modulation of structural equilibria. The principles uncovered in this study can be extended to the design of tunable, multifunctional antimicrobial protein therapeutics.

\section{MATERIALS AND METHODS}

Identification of membrane-active sequences using an SVM classifier

ACS Paragon Plus Environment 
The amino acid sequences of the human chemokines XCL1, CXCL4, and CCL20 were screened for membrane active domains using a previously validated support vector machine (SVM) classifier trained to recognize membrane permeable helical sequences. ${ }^{18}$ We implemented a variable moving window along the full sequences of each protein to give individual segments a $\sigma$-score indicative of the degree of likelihood that the segment is a membrane active peptide. To visualize the localization of these scores along the main sequence, we created a probability map displaying the scores along the sequence for each window size used. For a given window size, the score given to each amino acid position was calculated by averaging the mean o-score of all the segments in which that amino acid appears. The resulting probability map was plotted as a window size vs amino acid position heat map, in which the intensity values correspond to the mean o-score, i.e. the probability that the segment can induce membrane permeability.

\title{
Mutagenesis, Expression, and Purification of Recombinant XCL1 Proteins
}

\author{
The Quikchange Site-directed mutagenesis kit (Stratagene) \\ was used to perform site-directed mutagenesis on the XCL1 pET28a \\ expression vector and the XCL1 pQE30 expression vector in order \\ to create the following XCL1 variants: CC3 (XCL1 V21C V59C), CC0
}


(XCL1 C11A C48A), XCL1 1-72 (truncated at residue 72), XCL1 R23A, XCL1 R43A, XCL1 W55D, and CC5 (XCL1 A36C A49C). Each protein was then expressed and purified as previously described. ${ }^{13}$, 17,54 XCL1 and CCL28 were also produced and purified using this pipeline. In brief, each of these proteins was then expressed recombinantly in BL21 DE3 E. Coli with pET28a or pQE30 expression vectors containing the sequence for $\mathrm{His}_{6}-\mathrm{SUMO}-\mathrm{XCL1}$ or XCL1 variant sequence, except CC5 (XCL1 A36C A49C), which contained His8 affinity tag. Cultures were grown in terrific broth containing $50 \mathrm{lg} / \mathrm{mL}$ kanamycin to an optical density of $0.5-0.7$ at $37^{\circ} \mathrm{C}$, at which point $1 \mathrm{mM}$ isopropyl-b-D-thiogalactopyranoside (IPTG) was used to induce protein expression. After induction, cultures were grown for $5 \mathrm{~h}$ at $37^{\circ} \mathrm{C}$ and cells were harvested by centrifugation and stored at $-80^{\circ} \mathrm{C}$. Following expression and harvesting, proteins were purified. Cell pellets were resuspended in $50 \mathrm{mM}$ sodium phosphate $(\mathrm{pH} 8.0), 300 \mathrm{mM}$ sodium chloride, $10 \mathrm{~mm}$ imidazole, $0.1 \% \quad(\mathrm{v} / \mathrm{v}) \quad \beta$-mercaptoethanol, and $1 \mathrm{mM}$ phenylmethylsulfonyl fluoride (PMSF). Cells were then lysed by three passages through a French press and lysates were centrifuged at $12000 \mathrm{~g}$ for $20 \mathrm{~min}$ to collect inclusion bodies. Soluble fractions and resuspended inclusion bodies were incubated with $\mathrm{Ni}^{2+}-\mathrm{NTA}$ resin (Qiagen) for 1 hour at room temperature. Columns were rinsed and proteins were eluted with 6M guanidinium chloride, $50 \mathrm{mM}$ sodium phosphate (pH 7.4), $300 \mathrm{mM} \mathrm{NaCl}, 500 \mathrm{mM}$ 
imidazole, $0.2 \%$ sodium azide, and $0.1 \%$ (v/v) $\beta$-mercaptoethanol. Infinite dilution refolding in $20 \mathrm{mM}$ Tris ( $\mathrm{pH} 8.0$ ), $200 \mathrm{mM} \mathrm{NaCl}$, $10 \mathrm{mM}$ cysteine, and $0.5 \mathrm{mM}$ cystine, was performed for eluted proteins, followed by incubation at room temperature overnight with gentle stirring. The refolded protein solutions were concentrated to $\sim 50 \mathrm{~mL}$ and the fusion tag was removed by overnight Cleavage with ULP1 protease (His 6 -SUMO tag) or TEV protease (His8 tag). The tag was separated from the protein of interest by either cation exchange chromatography on SP Sepharose Fast Flow resin (GE Healthcare UK Ltd.) or reverse nickel chromatography using Ni2+-NTA resin (Qiagen) as above. Lastly, proteins were purified by reverse-phase high-performance liquid chromatography with a C18 column. Protein samples were then frozen and lyophilized. Sample identities, purities, and homogeneities were confirmed by matrix-assisted laser desorption ionization time-of-flight (MALDI-TOF) spectroscopy. Note that because the TEV system was used for CC5, the N-terminus of this protein was non-native, missing the N-terminal Vall.

\section{Liposome Preparation for SAXS Experiments}

Liposomes were prepared for SAXS experiments as previously described ${ }^{19},{ }^{34}$. In brief, lyophilized phospholipids DOPG (1,2dioleoyl-sn-glycero-3-[phospho-rac-(1-glycerol)]), DOPE (1,2dioleoyl-sn-glycero-3-phosphoethanolamine), DOPC (1,2-dioleoyl- 
sn-glycero-3-phosphocholine), and DOPS (1,2-dioleoyl-sn-glycero3-phospho-L-serine) purchased from Avanti Polar Lipids were dissolved in chloroform at $20 \mathrm{mg} / \mathrm{mL}$ to produce individual lipid stock solutions. Model membrane lipid compositions were prepared from the lipid stock solutions as mixtures at specified molar ratios. Each lipid mixture was subsequently evaporated under nitrogen and then desiccated overnight under vacuum to form a dry lipid film, which was resuspended in aqueous $140 \mathrm{mM} \mathrm{NaCl}, 10 \mathrm{mM}$ HEPES ( $\mathrm{pH}$ 7.4) to a concentration of $20 \mathrm{mg} / \mathrm{mL}$. Lipid suspensions were incubated overnight at $37^{\circ} \mathrm{C}$, sonicated until clear, and then extruded through a $0.2 \mu \mathrm{m}$ pore size Anopore membrane filter (Whatman) to form SUVs.

\section{SAXS Experiments with Model Membranes}

Methods used for SAXS experiments and data fitting were based around those that have been previously described ${ }^{30,} 33-34,55$. Lyophilized proteins (WT XCL1 and XCL1 variants, i.e. CC3 (XCL1 V21C V59C), CC5 (XCL1 A36C A49C), CC0 (XCL1 C11A C48A), XCL1 1-72 (truncated at residue 72), XCL1 R23A, XCL1 R43A, and XCL1 W55D) were solubilized in aqueous $140 \mathrm{mM} \mathrm{NaCl}, 10 \mathrm{mM} \mathrm{HEPES} \mathrm{(pH} \mathrm{7.4)} \mathrm{and}$ mixed with SUVs at $\mathrm{P} / \mathrm{L}$ of $1 / 2$ and $1 / 1$. Samples were hermetically sealed into quartz capillaries (Hilgenberg GmbH, Mark-tubes) for SAXS measurements taken at the Stanford Synchrotron Radiation Lightsource (SSRL, beamline 4-2) using monochromatic X-rays with 
an energy of $9 \mathrm{keV}$. The scattered radiation was collected using a DECTRIS PILATUS3 $X 1 M$ detector (pixel size, $172 \mu \mathrm{m}$ ) and the resulting 2D SAXS powder patterns were integrated using the Nika $1.50^{56}$ package for Igor Pro 6.31 and FIT2D. ${ }^{57}$ Using Origin Lab software, the integrated scattering intensity $I(Q)$ was plotted against $Q$. Ratios of the measured peak positions (Qmeasured) were compared with those of permitted reflections for different crystal phases to identify the phase(s) present in each sample. A linear regression through points corresponding to the peaks was used to calculate the lattice parameter, a, of each identified cubic phase. For a cubic phase, each peak is represented by a point with coordinates of the assigned reflection (in terms of Miller indices $h, k, I)$ and $Q_{\text {measured. }}$ For a cubic phase, $Q=(2 \pi / a) \sqrt{ }\left(h^{2}+k^{2}+l^{2}\right)$. Therefore, the slope of the regression $(m=2 \pi / a)$ of $Q_{\text {measured }}$ Vs. $V\left(h^{2}+k^{2}+l^{2}\right)$ can be used to calculate a. Similarly, for a lamellar phase, each peak is represented by a point with coordinates of the order of the reflection, $n$, and $Q_{\text {measured }}$ with the relation $Q=2 \pi n / d$. In this case, the slope $(m=2 \pi / d)$ of the regression of $Q_{\text {measured }}$ VS. $n$ is used to determine the periodic spacing, $d$.

\section{Candida Killing Assays}

Candida killing assays were performed in a similar manner to that which has been previously described ${ }^{11}$. ${ }^{45}$. In brief, yeast peptone 
dextrose (YPD) medium was used to culture a single clone of $C$. albicans strain CAF2-1 for $16-20$ hours at $30{ }^{\circ} \mathrm{C}$ and $250 \mathrm{rpm}$. Candida cultures were washed twice with low-salt buffer, $1 \mathrm{mM}$ potassium phosphate buffer (PPB). Washed cultures were diluted to $10 \mathrm{~mL}$ stock at final concentration of $\sim 5 \times 10^{4} \mathrm{cells} / \mathrm{mL} \mathrm{cell} / \mu \mathrm{L}$ in 1 mM PPB, pH 7.0, WT XCL1 and positive control chemokine CCL28 in lyophilized powder form were resuspended to a final concentration of $400 \mu \mathrm{M}$ in $1 \mathrm{mM} \mathrm{PPB}$ and stored at $-20{ }^{\circ} \mathrm{C}$ in $50 \mu \mathrm{L}$ aliquots. Proteins were serially diluted and mixed 1:1 with the Candida stock at $\sim 5 \times 10^{4} \mathrm{cells} / \mathrm{mL}$ to a total volume of $100 \mu \mathrm{L}$ in a 96-well plate. We performed a negative control (no protein) with vehicle treatment. After incubation of the 96-well plates at room temperature for $2 \mathrm{~h}$ with gentle shaking, appropriate dilutions of the suspensions were plated on YPD agar plates and incubated at 30 ${ }^{\circ} \mathrm{C}$ for 48 hours. Colonies were then counted, and viability was calculated as a percentage of the colony number of the no-protein control. Each protein condition was tested in triplicate, and assays were performed at least in duplicate.

\section{AUTHOR INFORMATION}

\section{Corresponding Author}

*Brian F. Volkman: bvolkman@mcw.edu

ACS Paragon Plus Environment 


\section{Author Contributions}

AFD, MWL, ARH, GCLW, and BFV conceived and planned experiments. AFD produced and purified proteins, analyzed data, prepared manuscript figures, and wrote the manuscript. MWL, JdA, AFD, and EYL performed SAXS experiments and MWL, JdA, and EYL analyzed data. JdA screened chemokine sequences using the machine-learning classifier and analyzed results. MWL and JdA assisted in manuscript writing, figure preparation, and revision. JH performed in vitro Candida assays and both JH and ARH analyzed results and assisted in manuscript revision. All authors have given approval to the final version of the manuscript.

\section{Competing interests}

B.F.V. has ownership interests in Protein Foundry, LLC.

\section{ACKNOWLEDGMENTS}

This work was supported in part by National Institutes of Health Grants R01 AI058072 and R01 AI120655 (to B.F.V.), K08 DE026189 (to A.R.H.), and F30 CA236182 (to A.F.D). A.R.H. was also supported by the Children's Hospital of Wisconsin Research Institute. A.F.D. is a member of the NIH supported (T32 GM080202) Medical Scientist Training Program at the Medical College of Wisconsin (MCW). J.d.A. is supported by the National Science Foundation Graduate Research Fellowship under Grant No. DGE1650604. M.W.L. and G.C.L.W. are supported by NIH R01AI052453, NSF DMR1808459, and the National Psoriasis Foundation. E.Y.L. acknowledges support from the Systems and Integrative Biology Training Program (T32GM008185), the Medical Scientist Training 
Program (T32GM008042), and the Dermatology Scientist Training

Program (T32AR071307) at UCLA, and an Early Career Research

Grant from the National Psoriasis Foundation.

\section{ASSOCIATED CONTENT}

\section{Supporting Information Available:}

Supplemental Figure 1. SAXS spectra for SUV-only control samples.

Supplemental Figure 2. SAXS spectra for protein-only control samples.

Supplemental Figure 3. SAXS spectra for XCL1 variant panel incubated with mammalian cell-like membranes.

This material is available free of charge via the internet at http://pubs.acs.org.

\section{REFERENCES}

1. Shai, Y., Mechanism of the binding, insertion and destabilization of phospholipid bilayer membranes by alphahelical antimicrobial and cell non-selective membrane-lytic peptides. Biochim Biophys Acta 1999, 1462 (1-2), 55-70. 2. Zasloff, M., Antimicrobial peptides of multicellular organisms. Nature 2002, 415 (6870), 389-95.

3. Wolf, M.; Moser, B., Antimicrobial activities of chemokines: not just a side-effect? Front Immunol 2012, 3, 213. 4. Crawford, M. A.; Margulieux, K. R.; Singh, A.; Nakamoto, R. K.; Hughes, M. A., Mechanistic insights and therapeutic 
opportunities of antimicrobial chemokines. Semin Cell Dev Biol 2019, 88, 119-128.

5. Nguyen, L. T.; Vogel, H. J., Structural perspectives on antimicrobial chemokines. Front Immunol 2012, 3, 384.

6. Heselpoth R.D., S. S. M., Linden S.B., Mitchell M.S., Nelson D.C., Enzybiotics: Endolysins and Bacteriocins. In Bacteriophages, Harper D., A. S., Burrowes B., McConville M., Ed. Springer, Cham: 2018.

7. Yung, S. C.; Murphy, P. M., Antimicrobial chemokines. Front Immunol 2012, 3, 276.

8. Crawford, M. A.; Fisher, D. J.; Leung, L. M.; Lomonaco, S.; Lascols, C.; Cannatelli, A.; Giani, T.; Rossolini, G. M.; Doi, Y.; Goodlett, D. R.; Allard, M. W.; Sharma, S. K.; Khan, E.; Ernst, R. K.; Hughes, M. A., CXC Chemokines Exhibit Bactericidal Activity against Multidrug-Resistant Gram-Negative Pathogens. MBio 2017, 8 (6).

9. Yang, D.; Chen, Q.; Hoover, D. M.; Staley, P.; Tucker, K. D.; Lubkowski, J.; Oppenheim, J. J., Many chemokines including CCL20/MIP-3alpha display antimicrobial activity. J Leukoc Biol 2003, 74 (3), 448-55.

10. Auerbach, D. J.; Lin, Y.; Miao, H.; Cimbro, R.; Difiore, M. J.; Gianolini, M. E.; Furci, L.; Biswas, P.; Fauci, A. S.; Lusso, P., Identification of the platelet-derived chemokine CXCL4/PF-4 as a broad-spectrum HIV-1 inhibitor. Proc Natl Acad SCi U S A 2012, 109 (24), 9569-74.

11. Hieshima, K.; Ohtani, H.; Shibano, M.; Izawa, D.; Nakayama, T.; Kawasaki, Y.; Shiba, F.; Shiota, M.; Katou, F.; Saito, T.; Yoshie, $0 .$, CCL28 has dual roles in mucosal immunity as a chemokine with broad-spectrum antimicrobial activity. J Immunol 2003, 170 (3), 1452-61.

12. Burkhardt, A. M.; Tai, K. P.; Flores-Guiterrez, J. P.; Vilches-Cisneros, N.; Kamdar, K.; Barbosa-Quintana, O.; ValleRios, R.; Hevezi, P. A.; Zuniga, J.; Selman, M.; Ouellette, A. J.; Zlotnik, A., CXCL17 is a mucosal chemokine elevated in idiopathic pulmonary fibrosis that exhibits broad antimicrobial activity. J Immunol 2012, 188 (12), 6399-406.

13. Nevins, A. M.; Subramanian, A.; Tapia, J. L.; Delgado, D. P.; Tyler, R. C.; Jensen, D. R.; Ouellette, A. J.; Volkman, B. F., A Requirement for Metamorphic Interconversion in the Antimicrobial Activity of Chemokine XCL1. Biochemistry 2016, 55 (27), 3784-93.

14. Murzin, A. G., Metamorphic Proteins. Science 2008, 320, 1725-1726.

15. Dishman, A. F.; Volkman, B. F., Unfolding the Mysteries of Protein Metamorphosis. ACS Chem Biol 2018, 13 (6), 1438-1446. 16. Kuloglu, E. S.; McCaslin, D. R.; Markley, J. L.; Volkman, B. F., Structural rearrangement of human lymphotactin, a C 
chemokine, under physiological solution conditions. J Biol Chem $2002,277(20), 17863-70$.

17. Tuinstra, R. L.; Peterson, F. C.; Kutlesa, S.; Elgin, E. S.; Kron, M. A.; Volkman, B. F., Interconversion between two unrelated protein folds in the lymphotactin native state. Proc Natl Acad SCi US A 2008, 105 (13), 5057-62.

18. Lee, E. Y.; Fulan, B. M.; Wong, G. C.; Ferguson, A. L., Mapping membrane activity in undiscovered peptide sequence space using machine learning. Proc Natl Acad Sci U S A 2016, 113 (48), $13588-13593$.

19. Hu, K.; Schmidt, N. W.; Zhu, R.; Jiang, Y.; Lai, G. H.; Wei, G.; Palermo, E. F.; Kuroda, K.; Wong, G. C.; Yang, L., A critical evaluation of random copolymer mimesis of homogeneous antimicrobial peptides. Macromolecules 2013, 46 (5), 1908-1915. 20. Matsuzaki, K.; Sugishita, K.; Ishibe, N.; Ueha, M.; Nakata, S.; Miyajima, K.; Epand, R. M., Relationship of membrane curvature to the formation of pores by magainin 2. Biochemistry $1998,37(34), 11856-63$.

21. Spaar, A.; Munster, C.; Salditt, T., Conformation of peptides in lipid membranes studied by $x$-ray grazing incidence scattering. Biophys J 2004, 87 (1), 396-407.

22. Yang, L.; Harroun, T. A.; Weiss, T. M.; Ding, L.; Huang, H. W., Barrel-stave model or toroidal model? A case study on melittin pores. Biophys J 2001, 81 (3), 1475-85. 23. Tang, M.; Waring, A. J.; Hong, M., Phosphate-mediated arginine insertion into lipid membranes and pore formation by a cationic membrane peptide from solid-state NMR. J Am Chem Soc 2007, 129 (37), 11438-46.

24. Saiman, L.; Tabibi, S.; Starner, T. D.; San Gabriel, P.; Winokur, P. L.; Jia, H. P.; McCray, P. B., Jr.; Tack, B. F., Cathelicidin peptides inhibit multiply antibiotic-resistant pathogens from patients with cystic fibrosis. Antimicrob Agents Chemother 2001, 45 (10), 2838-44.

25. Kalfa, V. C.; Jia, H. P.; Kunkle, R. A.; McCray, P. B., Jr.; Tack, B. F.; Brogden, K. A., Congeners of SMAP29 kill ovine pathogens and induce ultrastructural damage in bacterial cells. Antimicrob Agents Chemother 2001, 45 (11), 3256-61.

26. Yu, Y.; Vroman, J. A.; Bae, S. C.; Granick, S., Vesicle budding induced by a pore-forming peptide. J Am Chem Soc 2010, 132 (1), 195-201.

27. Gelbart W.M., B.-S. A., Roux D. , Micelles, Membranes, Microemulsions, and Monolayers. Springer, New York, NY: 1994. 28. Schmidt, N. W.; Wong, G. C., Antimicrobial peptides and induced membrane curvature: geometry, coordination chemistry, and molecular engineering. Curr Opin Solid state Mater Sci 2013, 17 (4), 151-163. 
29. Schmidt, N. W.; Tai, K. P.; Kamdar, K.; Mishra, A.; Lai, G. H.; Zhao, K.; Ouellette, A. J.; Wong, G. C., Arginine in alphadefensins: differential effects on bactericidal activity correspond to geometry of membrane curvature generation and peptide-lipid phase behavior. J Biol Chem 2012, 287 (26), 2186672 .

30. Schmidt, N. W.; Mishra, A.; Lai, G. H.; Davis, M.; Sanders, L. K.; Tran, D.; Garcia, A.; Tai, K. P.; McCray, P. B.; Ouellette, A. J.; Selsted, M. E.; Wong, G. C., Criterion for amino acid composition of defensins and antimicrobial peptides based on geometry of membrane destabilization. I Am Chem SoC 2011, 133 (17), 6720-7.

31. Yang, L.; Gordon, V. D.; Trinkle, D. R.; Schmidt, N. W.; Davis, M. A.; DeVries, C.; Som, A.; Cronan, J. E., Jr.; Tew, G. N.; Wong, G. C., Mechanism of a prototypical synthetic membraneactive antimicrobial: Efficient hole-punching via interaction with negative intrinsic curvature lipids. Proc Natl Acad SCi U $S$ A 2008, 105 (52), 20595-600.

32. Lee, M. W.; Chakraborty, S.; Schmidt, N. W.; Murgai, R.; Gellman, S. H.; Wong, G. C., Two interdependent mechanisms of antimicrobial activity allow for efficient killing in nylon-3based polymeric mimics of innate immunity peptides. Biochim Biophys Acta 2014, 1838 (9), 2269-79.

33. Xiong, M.; Lee, M. W.; Mansbach, R. A.; Song, Z.; Bao, Y.; Peek, R. M., Jr.; Yao, C.; Chen, L. F.; Ferguson, A. L.; Wong, G. C.; Cheng, J., Helical antimicrobial polypeptides with radial amphiphilicity. Proc Natl Acad Sci U S A 2015, 112 (43), 1315560 .

34. Lee, M. W.; Han, M.; Bossa, G. V.; Snell, C.; Song, Z.; Tang, H.; Yin, L.; Cheng, J.; May, S.; Luijten, E.; Wong, G. C., Interactions between Membranes and "Metaphilic" Polypeptide Architectures with Diverse Side-Chain Populations. ACS Nano 2017, 11 (3), 2858-2871.

35. Lee, M. W.; Lee, E. Y.; Lai, G. H.; Kennedy, N. W.; Posey, A. E.; Xian, W.; Ferguson, A. L.; Hill, R. B.; Wong, G. C. L., Molecular Motor Dnml Synergistically Induces Membrane Curvature To Facilitate Mitochondrial Fission. ACS Central Science 2017, 3 (11), 1156-1167.

36. Silvestre-Roig, C.; Braster, Q.; Wichapong, K.; Lee, E. Y.; Teulon, J. M.; Berrebeh, N.; Winter, J.; Adrover, J. M.; Santos, G. S.; Froese, A.; Lemnitzer, P.; Ortega-Gómez, A.; Chevre, R.; Marschner, J.; Schumski, A.; Winter, C.; Perez-Olivares, L.; Pan, C.; Paulin, N.; Schoufour, T.; Hartwig, H.; González-Ramos, S.; Kamp, F.; Megens, R. T. A.; Mowen, K. A.; Gunzer, M.; Maegdefessel, L.; Hackeng, T.; Lutgens, E.; Daemen, M.; Von Blume, J.; Anders, H.-J.; Nikolaev, V. O.; Pellequer, J.-L.; Weber, C.; Hidalgo, A.; Nicolaes, G. A. F.; Wong, G. C. L.; 
Soehnlein, O., Externalized histone H4 orchestrates chronic inflammation by inducing lytic cell death. Nature 2019, 569 $(7755), 236-240$.

37. Schmidt, N.; Mishra, A.; Lai, G. H.; Wong, G. C., Argininerich cell-penetrating peptides. FEBS Lett 2010, 584 (9), $1806-$ 13 .

38. Schmidt, N. W.; Mishra, A.; Wang, J.; DeGrado, W. F.; Wong, G. C., Influenza virus A M2 protein generates negative Gaussian membrane curvature necessary for budding and scission. J Am Chem SoC 2013, $135(37), 13710-9$.

39. Shearman, G. C.; Ces, O.; Templer, R. H.; Seddon, J. M., Inverse lyotropic phases of lipids and membrane curvature. J Phys Condens Matter 2006, 18 (28), S1105-24.

40. Robbyn L. Tuinstra, F. C. P., E. Sonay Elgin, Adam J. Pelzek, and Brian F. Volkman, An Engineered Second Disulfide Bond Restricts Lymphotactin/XCL1 to a Chemokine-like Conformation with XCR1 Agonist Activity. Biochemistry 2007, 46, 2564-2573.

41. Fox, J. C.; Tyler, R. C.; Guzzo, C.; Tuinstra, R. L.; Peterson, F. C.; Lusso, P.; Volkman, B. F., Engineering Metamorphic Chemokine Lymphotactin/XCL1 into the GAG-Binding, HIV-Inhibitory Dimer Conformation. ACS Chem Biol 2015, 10 (11), 2580-8.

42. Peterson, F. C.; Elgin, E. S.; Nelson, T. J.; Zhang, F.; Hoeger, T. J.; Linhardt, R. J.; Volkman, B. F., Identification and characterization of a glycosaminoglycan recognition element of the C chemokine lymphotactin. J Biol Chem 2004, 279 (13), $12598-604$.

43. Volkman, B. F.; Liu, T. Y.; Peterson, F. C., Chapter 3. Lymphotactin structural dynamics. Methods Enzymol 2009, 461, 5170 .

44. Volkman, B. F.; Liu, T. Y.; Peterson, F. C., Chapter 3 Lymphotactin Structural Dynamics. In Chemokines, Part B, 2009; pp $51-70$.

45. Thomas, M. A.; He, J.; Peterson, F. C.; Huppler, A. R.; Volkman, B. F., The Solution Structure of CCL28 Reveals Structural Lability that Does Not Constrain Antifungal Activity. J Mol Biol 2018, 430 (18 Pt B), 3266-3282.

46. Yount, N. Y.; Waring, A. J.; Gank, K. D.; Welch, W. H.; Kupferwasser, D.; Yeaman, M. R., Structural correlates of antimicrobial efficacy in $\mathrm{IL}-8$ and related human kinocidins. Biochim Biophys Acta 2007, 1768 (3), 598-608.

47. Kennedy, J.; Vicari, A. P.; Saylor, V.; Zurawski, S. M.; Copeland, N. G.; Gilbert, D. J.; Jenkins, N. A.; Zlotnik, A., A molecular analysis of NKT cells: identification of a class-I restricted $\mathrm{T}$ cell-associated molecule (CRTAM). J Leukoc Biol $2000,67(5), 725-34$. 
48. Kennedy, J.; Rossi, D. L.; Zurawski, S. M.; Vega, F., Jr.; Kastelein, R. A.; Wagner, J. L.; Hannum, C. H.; Zlotnik, A., Mouse IL-17: a cytokine preferentially expressed by alpha beta TCR + CD4-CD8-T cells. J Interferon Cytokine Res 1996, 16 (8), $611-7$.

49. Puel, A.; Cypowyj, S.; Bustamante, J.; Wright, J. F.; Liu, L.; Lim, H. K.; Migaud, M.; Israel, L.; Chrabieh, M.; Audry, M.; Gumbleton, M.; Toulon, A.; Bodemer, C.; El-Baghdadi, J.; Whitters, M.; Paradis, T.; Brooks, J.; Collins, M.; Wolfman, N. M.; Al-Muhsen, S.; Galicchio, M.; Abel, L.; Picard, C.; Casanova, J. L., Chronic mucocutaneous candidiasis in humans with inborn errors of interleukin-17 immunity. Science 2011, 332 $(6025), 65-8$.

50. Ferreiro, D. U.; Komives, E. A.; Wolynes, P. G., Frustration, function and folding. Curr Opin Struct Biol 2018, $48,68-73$.

51. Ferreiro, D. U.; Komives, E. A.; Wolynes, P. G.,

Frustration in biomolecules. Q Rev Biophys 2014, 47 (4), 285363.

52. Jenik, M.; Parra, R. G.; Radusky, L. G.; Turjanski, A.; Wolynes, P. G.; Ferreiro, D. U., Protein frustratometer: a tool to localize energetic frustration in protein molecules. Nucleic Acids Res 2012, 40 (Web Server issue), W348-51. 53. Romero, P. A.; Arnold, F. H., Exploring protein fitness landscapes by directed evolution. Nat Rev Mol Cell Biol 2009, 10 $(12), 866-76$.

54. Fox, J. C.; Nakayama, T.; Tyler, R. C.; Sander, T. L.; Yoshie, O.; Volkman, B. F., Structural and agonist properties of XCL2, the other member of the C-chemokine subfamily. Cytokine 2015, 71 (2), 302-11.

55. Lande, R.; Lee, E. Y.; Palazzo, R.; Marinari, B.; Pietraforte, I.; Santos, G. S.; Mattenberger, Y.; Spadaro, F.; Stefanantoni, K.; Iannace, N.; Dufour, A. M.; Falchi, M.; Bianco, M.; Botti, E.; Bianchi, L.; Alvarez, M.; Riccieri, V.; Truchetet, M. E.; G, C. L. W.; Chizzolini, C.; Frasca, L., CXCL4 assembles DNA into liquid crystalline complexes to amplify TLR9mediated interferon-alpha production in systemic sclerosis. Nat Commun 2019, 10 (1), 1731. 56. Ilavsky, J., Nika: software for two-dimensional data reduction. J Appl Crystallogr 2012, 45, 324-328.

57. Hammersley, A. P., FIT2D: an Introduction and Overview. European Synchrotron Radiation Facility Internal Report: ESRF97HAO2T 1997. 
Table of Contents Graphic:

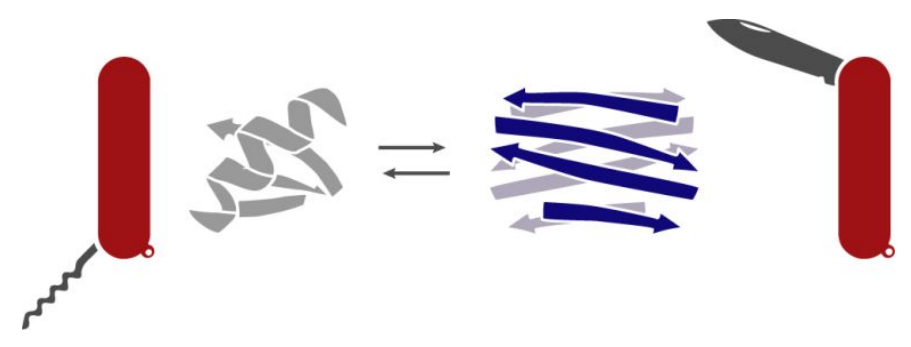

